(Aus der Prosectur des städtischen Spitals in Odessa).

\title{
Weitere Untersuchungen über die Veränderungen der Nervenzellen in verschiedenem Alter.
}

Von

Dr. med. $\boldsymbol{x}$. Mühlmann, Odessa.

Hierzu Tafel XII und XIII.

In meinen vorigen Untersuchungen ( 5 u. 6) habe ich nachzuweisen gesucht, dass in den menschlichen Nervenzellen schon in frühem Alter eine degenerative Erscheinung in Form von Fettpigmentbildung zu Tage tritt. Dabei habe ich im Anschluss an Rosin und gewissermassen auch an obersteiner als den Termin der anfänglichen Entwicklung des Fettpigmentes im Allgemeinen das Pubertătsalter genannt. Seit der Publication meiner letzten Arbeit auf der Versammlung Deutscher Naturforscher und Aerzte in Aachen im September 1900 habe ich die Studien weiter fortgesetzt und bin nunmehr zum Schluss gekommen, dass der Termin viel früher zurïckverlegt werden muss. Wie auf S. 152 der letzten Abhandlung entwickelt, beruhte die Feststellung des Pubertătsalters, als der Zeit der beginnenden Entwicklung des Fettpigmentes, auf folgende Thatsachen. Das Fettpigment tritt in den Nervenzellen des Erwachsenen in Form von hellgelbglănzenden Körnergruppen auf, die in einem bestimmten Theile des Zellleibes sitzen, ohne sich über das ganze Protoplasma zu zerstreuen, wie es bei der Fettmetamorphose der Fall ist; da bei den Kindern die mit Osmium geschwarzten Fettkörner keine festlocalisirte Lage im Zellleib hatten, sondern vereinzelt über das ganze Protoplasma zerstreut waren, da ausserdem die Körner bei frischer Untersuchung der kindlichen Nervenzellen den gelben Ton beinahe gänzlich verlieren und keinen Eindruck von Pigment, sondern direct von Fett machen, so nahm ich an, es handle sich dort um eine Fettmetamorphose, 
die durch die Infection, welcher die untersuchten Kinder unterlegen waren, verursacht wurde. Ich habe deshalb alle Kinder, bei welchen ich das Auftreten von Fettkörnchen in den Nervenzellen beobachtete, aus meiner Fettpigmentstatistik ausgeschlossen, und nur von dem Alter an das Auftreten des Fettpigments gerechnet, wo das letztere in Haufenform zur Beobachtung gelangt. Damals wurden der Untersuchung Nervenzellen von 6 Kindern im Alter von 1, 2, 3 und 8 Jahren ausgesetzt. Fettkörnchen wurden nur beim drei- und achtjahrigen Kinde gefunden. Das erste starb an Lyssa, das zweite an septische Scharlach. Beim ersten war das Pigment, wie erwähnt, hellglänzend und ziemlich regelmässig über die Zellen, sowohl aus dem Rückenmark als aus dem verlängerten Mark und den Spinalganglien zerstreut; dabei waren einzelne Zellen gänzlich von den Körnchen frei. Beim zweiten war allerdings in einzelnen Zellen eine geringe Anhäufung von Körnchen zu constatiren, die schwache gelbe Tinction derselben hielt mich jedoch davon ab, sie zum Fettpigment zu rechnen, umsomehr als solche Anhäufungen auch bei der Fettmetamorphose beobachtet werden können.

Der weiteren Untersuchung wurde das Centralnervensystem von 8 Kindern ausgesetzt, wobei derselbe Plan wie früher beibehalten wurde. Die Kinder waren 2, 3 (2 Kinder), 4, 8, 9, 12 und 15 Jabre alt. Diagnosen: Nepbritis, Hydrophobia, Combustio, Scarlatina und Typhus abd. Ausser der frischen Untersuchung der Prăparate wurden Stückchen von verschiedenen Theilen des Rückenmarks, vom verlängerten Mark (Boden des 4. Ventrikels), von der Centralwindung des Gehirns und von den Spinalganglien auf verschiedener Höhe in Flemming'scher Lösung gebracht resp. nach Marchi behandelt.

Fettkörner wurden bei allen Kindern ausser dem 2 jährigen und einem 3 jährigen Kinde gefunden; das erste starb an Nephritis acuta, das zweite an Typhus. Beim 8, 9, 12 und 15 jährigen war eine gelbe Tinction der Körner zu constatiren; ebenso gut konnten einzelne Häufchen von ihnen nach weisbar werden, dann wurde auch die goldgelbe Färbung stärker ausgesprochen. Bei dem einen dreijährigen, ebenso wie beim 4,8 und 9 jăhrigen Kinde waren die Fettkörner vereinzelt über die ganze Zelle unregelmässig zerstreut, wie auf Fig. $1 \mathrm{zu}$ sehen ist. Ehenso war dies hauptsächlich die morpbologische Vertretung der 
Weitere Untersuchungen üb. d. Veränderungen d. Nervenzellen etc. 233

Körnchen beim 12 jährigen und die vorwiegende beim 15 jährigen Knaben, obwohl bei beiden, besonders aber beim letzteren die Häufchenform ziemlich regelmässig beobachtet werden kounte.

Worum handelt es sich in den vorliegenden Făllen: um die normale Fettpigmenterscheinung oder um pathologische Fettmetamorphose? Ausser dem 4 jăhrigen Fall von Combustio handelt es sich in den übrigen Fällen um Infectionsprocesse.

Gegen die Fettmetamorphose spricht ganz besonders ein Umstand, den Virchow in den Vordergrund für die Charakteristik der Fettmetamorphose stellt, die Abwesenheit von einer weiteren Stufe derselben, einem Zellzerfall. Wir finden nicht eine zerstörte Zelle, wir finden keine Fettkörnerkugel. Wenn ich die Abwesenheit derselben in zwei Fällen bei meiner ersten Untersuchung einem Zufall zuschreiben konnte, so lässt die vermehrte Zahl der Beobachtungen jetzt keinem Zufall den Raum. Gegen die Bedeutung dieses Merkmales der Fettmetamorphose treten alle übrigen zurück: das Betroffensein des Kernes, die Ungleichmässigkeit der F'ettkörnchen, welche beide in unseren Fallen fehlen, was aber aus Rücksichten, die ich bereits hervorhob, von nebensächlicher Tragweite ist.

Wir dürfen also der Abwesenheit der gelben Tinction der Fettkörnchen in kindlichen Nervenzellen für die Analogie zwischen ihnen und erwachsenen keine Bedeutung zuschreiben und kommen dazu, der alten Virchow-Conheim'schen Auffassung zuzustimmen, dass die Nervenzellen von der pathologischen Fettmetamorphose gewissermassen eliminirt sind. Die Fettkörnchen der Kinder gehören zu denselben, welche bei Erwachsenen stärker goldgelb tingirt sind und in Häufchen den Nervenzelleib eingreifen. Sie stellen eine normale Erscheinung dar. Die morphologische Form ihres Auftretens beim Erwachsenen in Haufenform ist also für die Kinder nicht nöthig. Die gelbe Tinction ist bei Erwachsenen ganz besonders durch die massenhafte Anhäufung derselben stark ausgesprochen; bei der Betrachtung der einzelnen Körnchen kann man sich überzeugen, dass der Unterschied zwischen dem Kinde und dem Erwachsenen hierbezüglich minimal ist.

Fassen wir also die Ergebnisse dieser erweiterten Untersuchungen mit den früheren zusammen, müssen wir sagen, dass beim Menschen von den ersten Lebensjahren (nach meinen Beobachtungen vom dritten resp. vierten) an regel- 
massig in den Nervenzellen pigmentirte Fettkörnchen auftreten, die zuerst zerstreut, dann sich allmăhlich anhăufend das Protoplasma der Zelle besetzen und bereits im zweiten Lebensdecennium einen festen Platz im Zellraumeinnehmen. Mit dem Alter hăuft sich das Fettpigment im Centralnervensystem an, immer mehrZellen und immermehr Platz in den Zellen eingreifend, so dass bei Greisen die meisten Zellen mit den Körnchen gestopft sind und oft nur ein kleiner Protoplasmasaum in der Zelle davon freibleibt. Der Kern scheint in der Regel unlädirt zu sein.

Obwohl die geschilderte Erscheinung ein regelmässiges Vorkommen darstellt, hat man es erst in den letzten Jahren als solches kennen gelernt. Pilcz (1) hat im Jahre 1895 hauptsächlich frische Prăparate untersucht und mit der Natur der Erscheinung sich wenig beschäftigt; da er an einigen Zellen die Osmiumreaction probirte und die Körnchen dadurch dunkler gefärbt sah, so vermuthet er, es handle sich um Fett. Das erste Auftreten des Pigmentes, wie er es nennt, soll in den Spinalganglien im 6. Lebensjahr, im Rückenmark im 8. Jahr und im Gehirn im 20. Lebensjahr zur Beobachtung gelangen. Dies wurde von mir nicht bestätigt. Es lăsst sich kein Unterschied im Auftreten der Fettkörnchen zwischen den verschiedenen Theilen des Centralnervensystems constatiren. Ich habe beim dreijăhrigen Kinde dieselben sowohl im Rückenmark als in den Spinalganglien, als in den Pyramidenzellen der Hirnrinde gefunden. Dass Pilcz "das Pigment" in so frühem Alter noch nicht sehen konnte, darf Niemand wundern, wenn man berücksichtigt, dass er blos frische Objecte untersuchte. In diesen lassen sich die hellglänzenden Körnchen der kindlichen Nervenzellen im Zellleibe zerstreut sehr oft vermissen; sie waschen sich auch leicht ab. Für das Aufsuchen der Körnchen ist in diesem Fall die Osmiummethode unersetzbar: sie lässt kein Körnchen aus dem Auge fallen. Rosin (2), im Jahre 1896, trug zur Eruirung der Natur der Körnchen sehr bei, indem er die Fettnatur derselben hervorhob. Abgesehen von der Osmiumschwärzung ist von ganz besonderer Tragweite die Löslichkeit der Körnchen in Alkohol und Aether, worin man sich sehr leicht überzeugen kann, wenn 
man dünne Präparate nach 2-3 Tage langer Behandlung mit Alkohol und Aether in Osmium bringt: man erhält dann keine Schwärzung mehr. Ebensogut bewährt sich für den Beweis der fettigen Natur der Körnchen die Sudanfärbung, welche hierfür Rosin und Fenyvessy (3) vorschlugen. Allerdings konnte ich mich nicht im Unterschied überzeugen, welchen Sudan zwischen der Färbung der Körnchen und den Myelinscheiden bewirken soll; dass die Körnchen stärker roth gefärbt sind als die letzteren erklärt sich leicht aus dem Umstand, dass sie in Haufen liegen, vereinzelte Körnchen der Kinder färben sich mit Sudan ebenso, wie die Myelinscheiden der Nervenfasern. Indem die Lipochrombezeichnung einen chemischen Begriff darstellt, dessen Rechtfertigkeit für die Körnchen wissenschaftlich nicht festgestellt ist, enthalte ich mich dieser von Rosin vorgeschlagenen Bezeichnung derselben, umsomehr als dadurch den Körnchen eine bestimmte biologische Bedeutung zugeschrieben wird, mit welcher ich nicht einverstanden bin. Ich ziehe die von mir bereits angewandte Bezeichnung Fettpigment oder einfach Fett vor, um die Forschung durch zweifelhafte Terminologie nicht zu hemmen.

Die Entwickelung des Pigmentes in verschiedenen Altersstufen hat Rosin wenig interessirt; von jungen Individuen hat er blos zwei untersucht: ein 14 Monate altes und ein 17 jahriges. Die Nervenzellen des ersteren waren durch Osmium wie bestäubt, die des zweiten enthielten bereits reichliche Körnchen; indem er den Lipochrombefund beim ersteren als zweifelhaft hinstellt, sagt er in seiner ersten Arbeit, dass das Pigment vom Pubertătsalter, in der zweiten aber (mit Fenyvessy), dass es von der Kindheit an sich entwickelt, ohne neue Thatsachen zur Rechtfertigung dieser Behauptung anzuführen.

Das jüngste der von Obersteiner (4) untersuchten Individuen war 14 Jahre alt; von diesem Alter an stellte 0 bersteiner die Anwesenheit des Fettpigmentes in den Gliazellen der Mollecularschicht der menschlichen Hirnrinde fest.

Wenn wir eine Erscheinung feststellen, die sehr leicht zu beobachten ist und die regelmässig in jeder menschlichen Leiche, nach meinen Untersuchungen vom 3 . Lebensjahre an, zu finden ist, so entsteht unwillkürlich die Frage, warum man denn dieselbe bis zu den allerletzten Jahren vermass, und wenn man sie sah, man derselben keine Achtung schenkte. Dafür giebt es mehrere 
Ursachen. Erstens, werden zu den Untersuchungen normaler Nervenzellen der Säugethiere Kaninchen, Meerschweinchen gebraucht, bei welchen das Fettpigment, namentlich bei nicht sehr alten Thieren, beinahe fehlt. Zweitens, störte sehr die Untersuchung die Bezeichnung Pigment, welche den Körnchen zugeeignet war. Die Pigmente haben bis jetzt eine ziemlich stiefmütterliche Stellung in der Biologie; sollte jemand die Erscheinung in den Zellen gesehen haben, so glaubte er dadurch, dass er sagte, es wăre Pigment, die Frage nach der Natur derselben gelöst zu haben.

Aber am meisten schadeten der Untersuchung des Fettpigmentes die herrschenden Methoden der histologischen Untersuchung der Nervenpräparate. Die Labilität der frischen Objecte zwingt behufs Untersuchung der Nervenzellen dieselben künstlich $\mathrm{zu}$ fixiren und $\mathrm{zu}$ härten. $\mathrm{Zu}$ diesem $\mathrm{Zweck}$ gebraucht man Chromsăure, Sublimat etc., worauf die Präparate in Alkohol kommen, oder aber die Präparate werden direkt mit Alkohol behandelt, welcher die Fettkörnchen auflöst. Falls der zur Fixation oder zur Härtung gebrauchte Alkohol dies noch nicht gethan, beendigen die Auflösungder Fettkörnchen die üblichen Methoden des Einschlusses der Präparate in Celloidin oder in Paraffin, wobei sie vorerst in Aether oder in Xylol, Chloroform etc. kommen müssen. Auf diese Weise gelangt zur Untersuchung ein Präparat, welches kein Fett mehr enthält, and vom Fettpigment nur ein bald gelbliches, bald bräunliches Netz hinterbleibt, welches fălschlich für den natürlichen Befund gehalten wird; es ist dabei gar nicht der böse Wille des Beobachters Schuld, wenn er von "Pigment" in den Nervenzellen spricht. Besonders rückte Nissl's Methode diese normale Erscheinung in den Hintergrund. Nachdem die Prăparate mehrere Tage mit Alkohol bearbeitet werden, wodurch das Fett gänzlich eliminirt wird, werden sie mittelst Anilinfarben gefärbt, welche jeden Rest von Pigment dem Auge entwischen; auf diese Weise werden wir durch diese Methode, welche uns der Natur näher bringen will, von der Natur noch mehr entfernt. Die einzige Methode, welche die Möglichkeit bieten könnte, das Fettpigment näher kennen zu lernen, wäre diejenige von Marchi, weil dabei Osmiumsăure angewendet wird (die nachträgliche Behandlung mit Alkohol löst bekanntlich die erhaltene schwarze Farbung nicht mehr). Aber 
Weitere Untersuchungen üb. d. Veränderungen d. Nervenzellen etc. 237

Marchi's Methode wird gewöhnlich zu pathologischen Zwecken angewandt, und beinahe jeder Befund von scbwarzen Körnchen in den Zellen wird als pathologische Erscheinung gedeutet.

Meistens aber werden die Marchi'schen Präparate dick geschnitten und bei schwacher Vergrösserung betrachtet, weil die Behandlung die Feststellung von Fasern- resp. von Systemerkrankung bezweckt; auf Zellen wird dabei wenig geachtet.

Ich darf vielleicht nochmals daran erinnern, dass ich für die fettige Natur der Körnchen nicht allein die Osmiumschwärzung massgebend halte, sondern ganz besonders die Löslichkeit derselben in Alkohol und Aether. Was die Frage betrifft, ob nicht etwas anderes ausser Fett in den Zellen geschwärzt wird, so wird sie theilweise durch die Untersuchungen von Ledermann (7) theilweise durch diejenige von W lassak (8) in der Weise gelöst, dass nur Fett sich durch Osmiumsäure in jeder Beimischung (Chromsåure) schwarz färbt, während andere verwandte Substanzen sich dabei anders verhalten. Da die Hornschicht der Haut bei Osmiumbehandlung tiefschwarz gefärbt wird, so kann die Frage aufgestellt werden, ob nicht etwa Hornsubstanz durch Osmium geschwärzt wird: dann würde man noch an die Möglichkeit von Keratingehalt in den Nervenzellen denken müssen. Als ich aber die Haut vom Menschen, Kaninchen, Meerschweinchen und weisser Ratte mit Alkohol und Aether 3 Tage behandelte, ergab die nachträgliche Osmiumbehandlung keine Schwärzung mehr. Keratin löst sich weder in Alkohol noch in Aether; es wird also in der Haut nicht durch die Osmiumsäure geschwärzt. Die Schwärzung wird in der Haut wohl durch diffus gelöstes oder vertheiltes Fett verursacht.

Bezüglich der Bedeutung des Fettpigmentes finden wir in der Literatur keine Angaben. $\mathrm{Pilz}$ und Obersteiner sagen, dass darüber nichts bekannt ist. R o s in meint, dass die Erscheinung nichts pathologisches darstellt; dafür spricht auch die Bezeichnung Lipochrom.

Ich habe schon mehrmals den regressiven Charakter der Erscheinung hervorgehoben und will diesmal meinen Standpunkt etwas ausfübrlicher begründen.

Zunächst berief ich mich (5) auf die Thatsache des rückstăndigen Wachsthums des Gehirns im Allgemeinen. Diese Thatsache ist durch Gewichtsstudien an mehreren Tausenden von Gehirnen mit 
sorgfaltiger Wahl von Material ausreichend festgestellt. Das Gehirn erreicht sein maximales Gewicht bei Männern im 15. Lebensjahre (1490 gr.), bei Frauen im 14. Jahre (1345 gr.); darauf sinkt das Hirngewicht bis zum Alter (năheres finde man in Vierordt's Tabellen bis zum 25. Lebensjahre und bis zum Greisenalter bei mir). Warum hört das Hirnwachsthum früher auf als das Wachsthum des Organismus? Es müssen also Hemmungen vorhanden sein, die sein Wachsthum aufhalten. Die Berechnungen des relativen Hirnwachsthums zeigen, dass diese Hindernisse viel früher als im Pubertätsalter (14-15 Jahren) zu wirken anfangen. Wenn man das Wachsthum des Gehirns mit demjenigen des ganzen Körpers vergleicht, so erweisst sich, dass beim Neugeborenen das Hirngewicht $12,6 \%$ des Körpergewichtes ausmacht, beim zweijährigen Knaben 10,8\%, beim dreijăhrigen $9,5 \%$ u. s. w. immer weniger und weniger, so dass das Hirngewicht des 14 jährigen Knaben $4 \%$ und das des Erwachsenen $2 \%$ des Körpergewichtes beträgt. Im Vergleich mit dem Körperwachstbum wird das Hirnwachsthum schon von den ersten Lebensjahren an gehemmt. Hier ist nicht der Platz, die Ursache dieser Erscheinung zu suchen; darüber habe ich in meiner Alterstheorie das Năhere vorgebracht. Für uns ist jetzt wichtig die Thatsache selbst festzustellen.

Nachdem wir aus den makroscopischen Untersuchangen erfuhren, dass in der Entwickelung des Gehirns von den ersten Lebenstagen an Störungen auftreten, die, sich immer mehrend, im 14-15. Lebensjahr gănzlich das Hirnwachsthum stillstehen lassen und darauf sogar sein Gewicht herunterbringen, wollen wir auf mikroscopischem Wege nachsehen, worin sich diese Wachsthumshemmung aussert. Dass karyokinetische Theilungsfiguren der Nervenzellen selbst beim wachsenden Organismus nach der Geburt fehlen, ist eine bekannte Thatsache. Directe Theilung der Ganglienzellen des Menschen nach der Geburt hat ebensowenig Jemand beobachtet. Aber durch die Hemmung der Zellvermehrung kann man den Gang des Hirnwachsthums noch nicht erklären. 1)ie gehinderte Vermehrung kann eine Verlangsamung und Sistirung des Wachsthums erklăren. Wir sehen aber, dass das Hirngewicht rom 15. Lebensjahre an sinkt; es muss denn von dieser Zeit an unbedingt ein Verlust, eine Zerstörung von Substanz statthaben. 
Weitere Untersuchungen üb. d. Veränderungen d. Nervenzellen etc. 239

Die Untersuchung des Hirnwachsthums hat uns von selbst dazu gebracht, dass wir uns fragen müssen, ob nicht vielleicht die von uns beobachtete systematische Fettpigmententwickelung in den Nervenzellen eben jene mikroscopische Zeichen der Substanzzerstörung darstellen, welche wir behufs Erklärung des sinkenden Hirngewichtes suchen. Hierbezüglich müssen wir mit folgenden Thatsachen rechnen (dies werden also die zweite Reihe von Thatsachen sein, auf welche ich mich zur Begründung meiner Hypothese berufe).

An Stelle des Protoplasmas häuft sich in der Nervenzelle mit dem Wachstham des Organismus stets vermehrend Fett an, welches hier in derjenigen morphologischen Form vertreten ist, welche wir bei der Fettmetamorphose gewöhnt sind zu sehen, mit dem Unterschied, dass es hier an Pigment gebunden ist. Fett, eine leblose Substanz, tritt an Stelle des Protoplasmas, der lebenden Masse auf. Unter den Bestandtheilen des Protoplasmas der Nervenzelle spielen Eiweisskörper, als mit lebenden Eigenschaften bewaffnete Substanzen, dieselbe grosse Rolle, wie sonst im thierischen Körper, und wenn wir alle Gründe haben sie als gleichmässig im Protoplasma vertheilt sich vorzustellen, so müssen wir einsehen, dass das Fett hier auch an Stelle des Eiweisses auftritt. Obwohl die Nervenzelle beim Erwachsenen um etwas grösser ist, als beim Kinde, sehen wir doch, dass das Fettpigment ein unverhăltnissmässig immer viel grösseren Raum im Zellleib einnimmt. Auf Grund der von mir unternommenen Messungen der Spinalganglien nervenzellen konnte ich feststellen, dass, nachdem die Zelle eine gewisse Grösse beim Erwachenen erreicht hatte, sie mit dem fortschreitenden Alter des Individuums nicht mehr wächst. Der Fettpigmentgehalt der Zelle nimmt aber bis zum Greisenalter hin stets zu; das Fett tritt also ganz sicher an Stelle des Protoplasmas auf, welches aufgeht, zerstört wird. Aus diesem Grunde nannte ich den ganzen Process Fettpig mentmetamorphose.

Ich verstehe sehr wohl, dass das Sinken des Gewichtes irgend eines Organes aus ganz verschiedenen Ursachen zu Stande kommen kann; aber wozu brauchen wir alle mögliche Vermuthungen anzustellen, wo wir vor Augen den Zerstörungsprocess haben, welcher sehr leicht die regressive Entwickelung des Gehirnes erklären kann? 
Nach den Untersuchungen von W eis ba ch (9) enthält das kindliche Gehirn mehr Wasser, als das Gehirn des Erwachsenen (selbstverständlich ist dabei der Blutgehalt ausgeschlossen). Sollte das Wasser als selbständiger Bestandtheil der Zelle beim Kinde stärker vertreten sein, so muss es wohl eine wichtige Rolle spielen, wenn es im wachsenden und rege thätigen Gehirn reichlich vorhanden ist; seine Verminderung wird dann wohl keinen Fortschritt, sondern einen Rückschritt bedeuten. Sollte aber der Wassergehalt des kindlichen Gehirns desshalb grösser sein, weil das letztere mehr hygroscopische Bestandtheile enthält, so kann der Wasserverlust durch die Verminderung der Eiweisskörper auf Kosten des gebildeten Fettes zustande gebracht werden. In beiden Fällen haben wir mit einem regressiven Process zu thun; der erste Fall stellt aber eine Vermuthung dar, der weiter keine Thatsachen zur Stütze stehen, für die zweite haben wir unabweisliche mikroscopische Bilder.

Es könnte vielleicht noch eine Möglichkeit Platz greifen, dass die Verminderung des Hirngewichtes mit dem Wachsthum des Organismus durch die Vermehrung von Myelin auf Kosten der schwereren Substanzen verursacht wird. Dies würde aber ebensowenig die Thatsache umstürzen, dass ein Substanzverlust vorliegt und dass wir mit einem atrophischen Process zu thun haben. Geben wir aber einmal zu, dass das Gehirn im normalen Zustande regressive Vorgänge durchmacht, so müssen wir in den normalen Grenzen ebensngut die Möglichkeit regressiver Vorgänge in seinen Zellen zulassen.

Die Betrachtung der Fettpigmentbildung als eines regrestiven Processes könnte noch durch folgende Thatsache gerechtfertigt sein. Bis zur letzten Zeit war die fettige Pigmentirung der Nervenzellen als eine Greiseneigenthümlichkeit gut bekannt. Alle Histologen und Neurologen, welche das Pigment in den Greisenhirnen beobachteten, zweifelten im Mindestens nicht daran, dass man hierin mit einem Zeichen der senilen Atrophie der Zelle, also mit einem degenerativen Process zu thun hat. Leyden (10), der die fettige Pigmentirung bei einem Greise beobachtete, war über dessen Ausdehnung in den Rückenmarkszellen derart erstaunt, dass er die progressive Muskelatrophie der alten Menschen der Pigmentatrophie der Ganglienzellen der Vorderhörner zuschreiben möchte. Ebenso sind 
Weitere Untersuchungen üb. d. Veränderungen d. Nervenzellen etc. 241

die Beobachter, welche diese Erscheinung bei verschiedenen Kranken sahen, geneigt, an der Erkrankung der betreffenden Personen (paralysis progressiva, paralysis agitans u. A.) die Fettpigmentirung der Nervenzellen schuldig zu machen. Wir bringen somit nichts Neues hinein in der bereits vorhandenen Schätzung der fettigen Pigmentirung der Nervenzellen als eines degenerativen Processes: wir theilen blos mit, dass man dieser Erscheinung falscher Weise eine irgendwelche specifische krankheitserregende Eigenschaft zuzueignen Rechthat, weil sie nicht allein bei Greisen und nicht allein bei Kranken, sondern auch bei Leuten jeden Alters, in gewöhnlichem Sinne vollkommen normale n, beobachtet wird.

Wie reimen sich, wird vielleicht Jemand fragen, zwei so scheinbar entgegengesetzte Behauptungen: einerseits stellt die Fettpigmentirung einen degenerativen, atrophischen Process dar, andererseits kommt er im normalen Organismus vor? Das ist es ja, dass wir hier gerade mit einer derartigen atrophischen Erscheinung zu thun haben, welche dem normalen Organismus als unbedingtes Wachsthumspostulat eigen sind. Bekanntlich kommt im Organismus während des ganzen Lebens Verhornung, also Necrotisirung des Hautepithels vor, schleimige Metamorphose des Schleimhautepithels, Fettdegeneration des Talgdrüsenepithels, bei Frauen rege Zerstörung der Eizellen etc., kurz eine ganze Reihe von Processen, die ich im Allgemeinen zum dritten Stadium der wäbrend des Wachsthums zu beobachteten Atrophien zuzählte und als necrotisirende Atrophie chrakterisirte (11). Hierzu gehört also auch die Fettpigmentirung der Nervenzellen, welche somit eive normale Erscheinung regressiven Charakters darstellt.

Die Betrachtung der Fettpigmeutirung der Nervenzellen als eines degenerativen Processes könnte vom Standpunkte der herrschenden pathologischen Schule auf die Erwiderung stossen, dass ein degenerativer Process in sich den Begriff von einer gänzlichen Rückumwandlung, Zerstörung, Necrotisirung trägt; so führt die Fettmetamorphose zu einem völligen Zerfall der Zelle, die hyaline, amyloide Degeneration bringen das lebende Protoplasma gleichfalls um und vernichten die normale Function der Zelle, wogegen wir von der Nervenzelle diesbezüglich nichts 
wissen. Ob die Fettpigmentirung zur völligen Zerstörung der Zelle führt oder nicht, ist vorläufig wirklich unbekannt. Solange dies nicht bewiesen ist, müssen wir jedenfalls mit Lukjanow (12) daran erinnern, dass auch die Fettmetamorphose nicht überall einen gleichen Process darstellt; die Fettpigmentirung der Nervenzelle stellt einen Process sui generis dar, und es ist gar nicht nöthig ibn in die herrschende chablonmässige Gruppirung der regressiven Vorgånge hineinzuschieben, umsomehr als die Nervenzelle selbst sich in vieler Hinsicht sehr von den übrigen Körperzellen unterscheidet, in welchen die pathologischen Vorgänge sorgfältiger untersucht sind.

Man braucht deshalb keinen grösseren Werth einer anderen Entgegnung, die vielleicht eingeworfen wird, beimessen, dass bei den meisten Degenerationsprocessen und namentlich bei der. Fettmetamorphose der Kern der Zelle früher oder später in Mitleidenschaft gezogen wird, wogegen er hier eigenthümlicherweise unangetastet bleibt. Wenn wir uns daran erinnern, wie wesentlich der Kern der Nervenzelle von dem der anderen Zellen sich unterscheidet, dass er in morphologischer Hinsicht keinen Prototypus in den jeglichen Kernformen des vielzelligen Organisums hat, wenn wir erwăgen, wie verschieden das Protoplasma der Nervenzellen vom Protoplasma anderer Zellen in chemischer Beziehung reagirt, und zwar in dem Sinne, dass die Reactionen des Protoplasma der Nervenzellen in vielem den Reactionen der Kernstubstanzen anderer Zellen analog sind, indem sie basophile Körner (Nissl's) enthält und somit viel mehr Kernsubstanz vielleicht in sich birgt, als das Protoplasma anderer Zellen, werden wir nichts wunderbares darin finden, dass die Nervenzelle auf pathologische Einwirkung anders reagirt, als die sonstigen Körperzellen. Es ist wohl möglich, dass die Läsion der chromophilen Protoplasmakörner, welche hier unzweifelhaft stattfindet, indem bei älteren Personen beinahe der ganze Zellleib in den Process der fettigen Pigmentirung eingegriffen wird, der Läsion der Kerne anderer Zellen analog ist.

Ich brauche wohl kaum noch die Frage discutiren lassen zu müssen, ob wir hier nicht etwa mit einer Fettinfiltration zu thun haben. Die letztere stellt übrigens einen physiologischen Vorgang vor, wenn Fett an bestimmten Orten abgelagert wird oder wenn es transitorisch, wie z. B. manchmal in der Leber, 
Weitere Untersuchungen üb. d. Veränderungen d. Nervenzellen etc. 243

vorkommt, sonst ist der Process ebenso pathologisch wie die Fettmetamorphose und nach neuen Untersuchungen wird die Grenze zwischen beiden sehr schwer zu ziehen sein. In unserem Fall liegt ein ziemlich regelmässiger morphologischer Unterschied sowohl von der Fettinfiltration als von der Fettmetamorphose vor : erstens bei Erwachsenen die Lage des Fettpigmentes an ganz bestimmten Orten der Zelle, zweitens aber gewöhnlich die Gleichheit der Körnergrösse, welche sowohl bei der Fettmetamorphose, als namentlich bei der Fettinfiltration fehlt. Bei der ersteren werden oft ebenso kleine Fettkörnchen gefunden, wie bei der Fettpigmentirung, sie sind aber nie so regelmässig gleich an Grösse wie hier. Auch ist die Form der Fettpigmentkörner nicht geometrisch rund, wie bei den echten Fettprocessen; da wo die Fettpigmentkörnchen in Gruppen angehäuft sind, sind sie oft durch die Anlagerung aneinander eckig.

Was den Einwand betrifft, dass ein Degenerationsprocess eine Abschwächung resp. eine Vernichtung der Function postulirt, so wissen wir doch über die Function der Nervenzelle so wenig, dass es wirklich kaum lohnend ist diese Frage zu berühren. Wir wissen aber sehr gut, dass keine einzige Zelle im Organismus in so ausgedehnter Weise wie die Nervenzelle die Fähigkeit compensatorischer Thätigkeit besitzt. Es genügt desshalb zu wissen, dass nicht alle Zellen in den Zerstörungsprocess eingegriffen sind oder dass in der Zelle noch unverănderte Theile vorhanden sind, besonders aber dass der Kern mit dem Kernkörperchen unlädirt sind um zu verstehen, dass die regressiven Veränderungen einen hohen Grad der Ausbreitung erreichen können ohne Abschwächung der Function und sichtbare krankhafte Veränderungen in den Verrichtungen des Körpers unbedingt hervorzurufen. Diese Ansicht hat eine morphologische Stütze in der Thatsache, dass es wirklich Wunder erregen kann, wie unangetastet derjenige Theil der Nervenzelle aussieht, welcher von der Fettpigmentbildung verschont bleibt. Er mag noch so gross oder klein sein, er mag bis zu einem schmalen Ring, wie z. B. in der Fig. 3 bei einem 80 jährigen Individuum (die centrale Zelle) zu sehen ist, reducirt sein, er zeigt keine abnorme Veränderung, keine Vacuolisirung, keine sonstigen Zerklüftungen oder Hyalinisirung, die Farbenreactionen sind darin gut erhalten, kurz, es scheint, dass wir mit einem vollkommen gesunden Zellen- 
theil zu thun haben. Ebenso gut sieht der Kern aus. Ich konnte zwischen dem Kern der kindlichen und dem der senilen Nervenzellen keinen Unterschied sowohl im Verhalten zu Farbstoffen als in frischen Präparaten constatiren; die Körnelung desselben, welche bei den Kindern beobachtet wurde, rührte wahrscheinlich von der Wirkung des Infectionsprocesses her. Der Fettpigmentirungsprocess scheint schleichend von Mollecül zu Mollecül in Appositionsform, ohne Tendenz Theile zu überspringen, das Protoplasma der Nervenzelle zu zerstören. Von den zahlreichen Folgerungen, welche sich aus meinen Untersuchungen ziehen lassen, will ich zwei hervorheben.

Es wurde bereits erwähnt, dass einige Neuropathologen den Befund von fettiger Metamorphose der Ganglienzellen bei nervösen Kranken (progressiver Paralyse, Paralysis agitans, bei Systemerkrankungen) mit der Krankheit in ursächliche Beziehung bringen. Die Ergebnisse der obigen Untersuchungen zeigen, wie vorsichtig man bei derartigen Schlüssen sein muss. Es soll damit nicht gesagt sein, dass die Nervenzellen etwa nicht spezifisch durch die eine oder die andere pathologische Einwirkung erkranken können; es sei damit blos an besondere Vorsicht gemahnt, welche jeden einzelnen Fall individualisiren und vor Allem die Frage lösen lässt, inwiefern die pathologischen Verănderungen diesem oder jenem Alter als normale Erscheinungen eigen sind. Wir besitzen vorläufig noch kein genaues Kriterium, um zu sagen, wie hochgradig die Verănderungen in jedem einzelnen Alter sein müssen; unsere Untersuchungen ergeben blos eine allgemeine Vorstellung von der Sache, indem sie lehren, dass die Veränderungen beim Kinde schwach und mit dem Alter stärker werden. Man kann also sicher nur dann von specifisch-pathologischer Fettdegeneration sprechen, wenn man beim Kinde ebenso starke Fettmetamorphose findet, wie beim Erwachsenen. Beim Erwachsenen wird man dagegen dann von Anomalie sprechen können, wenn man in seinen Zellen gar nicht oder wenig Fettpigment finden wird, wie beim Kinde, oder wenn die Fettkörnchen bei frischer Untersuchung keine Goldgelbfärbung zeigen werden. Auf Grund des Osmiumpräparates wird man in Făllen starker Fettmetamorphose nie ein richtiges Urtheil in Bezug auf die Pathologie des Falles abgeben können. 
Weitere Untersuchungen üb. d Veränderungen d Nervenzellen etc. $24 \grave{b}$

Die andere Schlussfolgerung betrifft die biologische Bedeutung des Fettpigmentes. Wenn man bedetıkt, dass in Zellen des normalen Organismus systematische Verănderungen regressiver Natur von den ersten Lebenstagen bis zum Tode hin beobachtet werden, wenn man in Rücksicht zieht, dass diese degenerativen V'erănderungen die Zellen des centralen Nervensystems ergreifen, wird man wohl in Anbetracht der hervorragenden Rolle, welche das Nervensystem in der Wirthschaft des Organismus spielt, kaum Wunder nehmmen, dass ich mir auf Grund der vorgefübrten Beobachtungen eine weite Verallgemeinerung bezüglich der Ursache der senilen Atrophie und des Todes erlaubte. Eine specielle Besprechung dieser Frage begann ich bereits an einem anderen Orte (5). Indem ich hier mich mit dem Hinweis auf mein Buch beschrănken muss, will ich mir blos eine kurze deutlichere Formulirung meiner Ansicht vom Zusammenhang zwischen dem morphologisch regressiven Process in den Nervenzellen und den Functionen des Organismus gestatten.

Dank der hochgradigen compensatorischen Fähigkeit des Protoplasmas der Nervenzelle übtder degenerative Vorgang, welcher von den ersten Lebensjahren sich in demselben beobachten lasst, keinen scbädlichen Einfluss auf die Verrichtungen des Organismus aus. Auf diese Weise vermag die Compensationsthatigkeit des unversehrten ProtoplasmasdieAeusserung derpathologischen Wirkung der Fettmetamorphose gewissermaassen zu verschleiern. Mit dem Masse aber, als die Zahl der Fettkörnchen sich in den Nervenzellen vermehrt und die Zahl der betroffenen Zellen vergrössert wird, vermindert sich sowohl die Masse des activen Protoplasmas und damit in Zusammenhang seine compesatorische Thatigkeit, so dass schliesslich die pathologische Seite die Oberhand nimmt und in der Functionsausübung des Organismus sich Defecte merkbar machen, welche das böhere Alter charakterisiren. Der stets weiter fortschreitende Degenerationsprocess in den Zellen vermindert die functionelle Fähigkeiten des Organismus in immer höheremGrade, und wenndie Läsion in die wichtige 
Lebenscentra, in die Medulla oblongata stärker eingreift, komm t as Leben zu Ende.

An der Läsion der letzteren Centra kann man sich durch unmittelbare Beobachtung überzeugen: die Fig. 3 giebt ein Urtheil ab von der Fettpigmentmetamorphose, welche der Hypoglossuskern einer 80 jährigen Greisin erlitten hat. Analoge Fettpigmentphänomene kann man bei hohen Greisen im Vaguskern beobachten.

Vergleichshalber füge ich noch eine Abbildung von den Zellen aus derselben Hirnstelle bei einem 18jăhrigen Manne hinzu (Fig. 2). Wir treffen hier gerade die Mittelstufe zwischen dem kindlichen und dem Greisenbefund. Einerseits treten hier die Körnchen noch ziemlich zerstreut über den ganzen Zellleib auf und haufen sich noch nicht dicht an einer Stelle des Protoplasmas an, wie es nach dem 20. Lebensjahre meist der Fall ist. Andererseits sind sie zwar auf dem ganzen Querschnitt der Zelle zu sehen, sie sind aber viel dichter an einem Orte aneinander gedrängt, als es beim Kinde der Fall ist.

\section{Literatur.}

1. Pilcz, Beitrag zur Lehre von der Pigmententwicklung in den Nervenzellen. Arbeiten aus dem Institut von Obersteiner Bd. III. 1895.

2. Rosin, Ein Beitrag zur Lehre vom Bau der Ganglienzellen. D. m. W. 1896. No. 31 .

3. Rosin und Fenyvessy, Ueber das Lipochrom der Nervenzellen. Virchow's A rchiv. 1900.

4. O b e r s t e i n ex, Zur Histologie der Gliazellen der Mollecularschichte der Grosshirnrinde. Arbeiten aus dem Institut von Obersteiner. 1900.

5. M ü h I m a n n, Ueber die Ursachen des Alters. Grundzüge der Physiologie des Wachsthums. Wiesbaden 1900.

6. I $\mathrm{d}$ e $\mathrm{m}$, Ueber die Veränderungen der Nervenzellen in verschiedenem Alter. Verhandl. der Deut. Pathol. Gesellschaft Bd. III. 1901. Refer. in Central. für Allg Pathol und pathol. Anat. 1900.

7. Ledermann, Ueber die Osmirung der normalen Haut. Berl klin. Woch. 1892.

8. Wlassak, Die Herkunft des Myelins. Arch. f. Entwickelungsmechanik Bd. VI. 1898.

9. W e i s b a ch, Cit. nach Hammarsten. Lehrbuch d. phys. Chemie. 1899.

10. L e y d e n, Klinik der Rückenmarkskrankheiten. 1875.

11. Mühlmann, Atrophie und Entwickelung. Deut. med. Wochen. No, 41. 1900 . 
Franz Weidenreich: Das Gefässsystem đer menschlichen Milz. 247

12. Lukjanow, Grundzuge der allgemeinen Pathologie der Zelle. Warschan 1890 (es giebt auch eine deutsche Uebersetzung).

\title{
Erklärnng der Abbildungen auf Tafel XII nnd XIII.
}

Fig. 1. Theil eines lumbalen Spinalganglions eines 3 jährigen Knaben . Flemming. Saphranin. Leitz Oc. 3. Ob. 7. Die durch Osmiumsäure schwarz gefärbten Fettkörnchen sind regelmässig über die ganze Zelle zerstreut. Viele Zellen sind noch von der Fettpigmentbildung frei.

Fig. 2. Aus dem Hypoglossuskern eines 18 jährigen jungen Mannes. Flemming. Leitz Oc. 3. Ob. 7. In einzelnen Zellen sieht man bereits eine Anhäufung der Fettkörnchen; im übrigen Zellraum sind ausserdem vereinzelte Fettkörnchen, wie beim Kinde, sichtbar.

Fig. 3. Der Hypoglossuskern einer 80 jährigen Frau. Flemming. Saphranin. Leitz Oc. 4 Ob. 4 . In der Zeichnung ist der zellenlose Zwischenraum zwischen der Ependymauskleidung und der Zellenschicht abgekürzt dargestellt. Einige Zellen sind vollständig von den Körnchenhaufen ansgefullt und nur ein geringer Protoplasmasaum bleibt davon frei. Es giebt hier keine körschenfreie Zellen mehr.

(Aus dem anatomischen Institut in Strassburg).

\section{Das Gefässsystem der menschlichen Milz.}

\author{
Von \\ Dr. Franz Weidenreloh, \\ Assistent am anatomischen Institut. \\ Hierzu Tafel XIV und XV and 1 Textfigur.
}

\section{Inhaltsverzeichniss.}

Einleitung. Seite

Untersuchungsmethoden . . . . . . . . . . . . . . . . 250

Begriffsbestimmung . . . . . . . . . . . . . . . . 254

I. Zurückleitende Gefässbahnen . . . . . . . . . . . . . . 255

A. Balken- und Pulpavenen . . . . . . . . . . . . . 255

B. Milzsinus . . . . . . . . . . . . . . . . . . . 256

Literatur . . . . . . . . . . . . . . . . 256

a) Endothelzellen . . . . . . . . . . . . . . 257

Literatur . . . . . . . . . . . . . . . . . 261

Kritische Besprechung der Literatur . . . . . . 263 